San Jose State University

From the SelectedWorks of Ted M. Coopman

May 24, 2009

\title{
Toward a Pervasive Communication Environment Perspective
}

Ted M. Coopman, San Jose State University 
Toward a Pervasive Communication Environment Perspective

\author{
Ted M. Coopman \\ Department of Communication Studies \\ Department of Television, Radio, Film, and Theatre \\ San José State University \\ One Washington Square \\ San José, CA 95192-0112 \\ tmcoopman@yahoo.com
}

A paper submitted to the Philosophy of Communication Division of the International Communication Association, annual convention 2009 


\begin{abstract}
In a world where pervasive communication technologies facilitate an increasing percentage of human interaction, the traditional dichotomy between face-to-face and mediated communication (especially computer mediated communication) obscures more than it illuminates. This has impacts for both teaching and research. To address this, I propose a holistic approach: Pervasive Communication Environment Perspective (PCE). Represented as a graphic model, PCE illustrates the circular flow of information and communication across mediums, channels, and individuals. This provides a conceptual tool with practical applications for teaching as well as research.
\end{abstract}




\section{Toward a Pervasive Communication Environment Perspective}

Since the advent of writing, every new communication technology has been lauded as the bright beginning of a new era or the end of civilization as we know it. As is often the case, innovations in communication usually result in some combination of the two extremes. Plato decried written language as something that would lead to intellectual laziness since people would no longer have to memorize. It is easy for us to laugh at the image of a toga-clad senior decrying "these dang kids and their new fangled writing," but similar arguments seem to arise whenever innovation appears. As humans, we naturally compare what is new to what we have known. We make judgments based on our own experiences, needs, and desires. When the public begins to adopt any new communication technology an inevitable and usually unfavorable comparison is made between it and "the gold standard" of communication; face-to-face interaction.

Humans have a "communication imperative" (Thurlow, Lengel, and Tomic, 2004) that drives us to maximize our communication satisfaction and interaction. We invariably circumvent any obstacles that interfere with our relational fulfillment. That is, it is not that technology affords or determines how we interact, but that we appropriate it to serve our own ends. (Thurlow and Brown, 2003). Most communication technology, from the printing press, to the telephone, to the Internet and text messaging is marked by humans bending it to serve basic communication needs and desires. This includes physical venues such as coffee houses or public squares that emerged as places that facilitate interaction (Alexander, Ishikawa, Silverstein, Jacobson, Fiksdahl-King, and Angel, 1977).

While there is certainly a degree of utility in compartmentalizing different aspects and types of communication in their relation to new or old media, the increasing pervasiveness of communication technologies and the trend toward the collapse of old and new media (often 
hyped as "convergence") into just "media," casts the continued demarcation of computer mediated versus non-computer mediated communication (CMC) into doubt as an analytical framework for understanding complex social interactions or cases.

It is certainly true that in many cases the continued study of specific channels of communication and their characteristics has value. This is not a call for a wholesale retreat from or rejection of thematic and sub-disciplinary specialties that concentrate on specific interaction venues, modes, or styles. These types of studies have and will continue to yield untold volumes of valuable knowledge and insight. However, I argue that the general demarcation between different modes when discussing broader phenomenon is in danger of obscuring our understanding of human communication processes. Moreover, as much as those academics who "get it" in terms of the impact of technology consider this central tendency of communication technologies to permeate everyday lives obvious, many of our colleagues do not.

There remains a strong bias toward face-to-face communication as distinct, preferential, and isolated from all other forms. That is, the dichotomy of face-to-face and everything else. Unfortunately, this is a view that can have negative ramifications if we as educators perpetuate this view to our students. This is a view that conflicts not only with student's lived experience, but also with the demands of life outside our educational institutions. At worse, it facilitates the perception of the academy as out of touch with "the real world." A basic unified perspective taking into account our more complex environment might be a tool to facilitate a more inclusive and complex understanding of our developing communication environment.

I begin by briefly discussing and contextualizing some of the literature on face-to-face versus Computer Mediated Communication (CMC). Next I will present my model of the pervasive communication environment (PCE). Finally, I discuss the analytic utility of this model 
and implications for its future use.

Anything You Can Do I Can Do Better: Aspects of Face-to-Face and Computer Mediated

\section{Communication}

Numerous studies have explored the advantages and disadvantages of CMC versus faceto-face communication. One of the earlier analyses (Bordia, 1997) synthesized the experimental literature examining face-to-face communication and CMC. Bordia found that CMC discussions took longer to accomplish the same amount of work and that in a given time frame and CMC participants made fewer remarks when compared to face-to-face communication. This is likely due to the mediated nature of online discussion. However, the number of remarks and task related comments were the same within the time-period used to complete a task. Moreover, there was no significant difference in the ability of these groups to successfully complete their tasks. CMC groups outperformed face-to-face groups on idea generation, the reduction of production blocking, and evaluation apprehension. Further, CMC groups were superior in their ability to scale up in size with no decrease in individual productivity. CMC groups showed a greater equality in participation and reduced normative social pressure. Where CMC fell short was in the area of social-emotional interaction. The unavailability of social cues and the extended time needed for $\mathrm{CMC}$ versus face-to-face communication made incidental talk less likely to occur. The lack of cues also resulted in an increase in uninhibited behavior in CMC groups, which depending on the behavior could be a positive or negative attribute of reduced normative social pressure.

Brandon and Hollinghead (1999) found CMC increased levels of student responsibility and levels of participation. Althaus (1997) noted that there were no significant demographic 
differences between the students who chose to participate in computer-mediated discussion (CMD) versus face-to-face discussions. His research also found that CMD appeared to be more egalitarian than face-to-face communication in terms of participation. Stewart, Shields \& Sen (1998) found that those who traditionally dominate face-to-face discussions, white males, tended to post more often and longer than women or non-whites. While Postem, Spears, \& Lea (2000) noted that out of context or face-to-face interaction had less an impact on emergent norms than the use of technology itself in groups. Ramirez, Walther, Burgoon, and Sunnafrank (2002) argued that "CMC facilitated people to seek information in new and unique ways and that rather than a tool that constrains information seeking, it can be seen as a way to offer unique manifestations of strategies available through other communication forms" (p.219). A combined meta-analysis and study by Benoit, Benoit, Milyo, and Hansen (2006) found no significant difference in learning between traditional and web-based formats and that learning and satisfaction are increasing gradually over time. And on it goes.

But how often is all action or interaction bifurcated between purely mediated and socalled unmediated parties? Moreover, even if we cede the superiority of face-to-face communication over all other forms (a claim I do not support) the reality is we are faced with a world that demands knowledge and understanding of combined forms of communication. It is possible that we will find (or already find) ourselves in a place where pure face-to-face interaction is more the exception than the rule?

How productive is it to draw clear distinctions between two primary modes of communication? Those who self-identify as Internet researchers readily discourse on Information and Communication Technologies (ICT), Computer Mediated Communication (CMC), Computer Mediated Discussion (CMD) as well as virtual anything (Papacharissi, 2005). Our 
focus on the impact of new technologies of communication led us to comparisons with face-toface communication as a separate and distinct phenomenon. This is perpetuated by textbooks that include a "new" chapter on technology as an added-value component for the latest edition (e.g. small groups AND use of technology in small groups), thus reinforcing the "other" and "also" of the technological or so-called virtual world.

As mentioned earlier, students are at once amused and confused by the conflicts between their lived experiences and the information they are given in our courses. Text messaging, social networking websites, laptops with wireless Internet access, instant messaging and email are not separate parts of their lives but part of what makes their lives work. Despite this, they often parrot back the inferiority of mediated communication as compared to face-to-face interaction. So their analysis rests on the proposition of a false choice between mediated and non-mediated communication for interaction.

A recent study (An \& Frick, 2006) of student preferences indicated that the lack of involvement and enthusiasm of instructors for $\mathrm{CMC}$ and teaching how to use it was a factor in their dissatisfaction. Moreover, the students surveyed placed more of a premium on speed and convenience than whether the communication was mediated or face-to-face. Rafaeli's (1988) early analysis of interactivity took a "medium independent" approach, which more accurately address lived experiences. That is, it is the actual exchange, not the medium in which it takes place that is key. As any who has faced a classroom full of students, physical co-location does not ensure interactivity!

Interpersonal networks under the impact of new technologies are transitioning from territorialized relationships rooted in physical space to "networked individualism" or "role-torole" relationships (Wellman, 2000). As computing has moved from a centralized to a dispersed 
activity, so have interpersonal relationships. Social capital is increased through the augmentation of physical interaction with interaction over the Internet, and "the ease with which CMC connects friends of friends can also increase the density of interconnections among clusters of network members within communities" (Wellman, 2001, p. 2032).

Kim and Ball-Rokeach (2006) conceptualized Communication Infrastructure Theory (CIT) as an ecological approach to assessing civic engagement. They combined all communication resources available, from interpersonal networks to mass media, as integral aspect of community storytelling and cohesion. Castells (1996) went as far as to declare a new age, the "network society," powered by the Internet and other new communication technologies. He stated, "networks are very old forms of human practice, but they have taken on a new life in our time by becoming information networks powered by the Internet” (Castells, 1996, p. 1). He pointed out that technology enabled networks, because of their flexibility and adaptability, have tremendous advantages as organizing tools in our fast changing political, social, cultural, and economic environment.

Walther, Gay, and Hancock (2005) addressed the dearth of empirical theory on communication technologies as a probable factor of the newness of the field of CMC. They argue that the importance of communication research on technology is grounded in its impact on "social, organizational, political, and relational aspects of our daily lives as other media such as television and the telephone have in the past (p. 652)." At issue is the central focus of the inquiry. For example, is it more about the organization or the technology? While it is certainly productive to examine the impacts of specific technologies on the communication process, when do we reach the point of diminishing returns? How much of this research is concerned with the novelty of an increasing less novel technology? When do the differences between CMC and 
face-to-face interaction become less significant than the communication act itself? Can we as researchers continue to divide the world up this way when much of the world doesn't perceive the divisions?

The February 2006 Wired magazine asked a number of technorati about the death of "cyberspace" as a term. William Gibson, who coined the term in the mid-1980s remarked, "I think cyberspace is past its sell-by, but the problem is that everything has become an aspect of, well, cyberspace" (Pang and Pescovitz, 2006, p. 39). Even the utility of the word "internet" (downgraded from Internet by pioneering CMC researcher Steve Jones) has been debated among internet and media scholars. A Pew study on The Strength of Internet Ties released concurrently as the Wired article observes;

"Once upon a time, the internet was seen as something special, available only to wizards and geeks. Now it has become part of everyday life. People routinely integrate it into the ways in which they communicate with each other, moving between phone, computer, and in-person encounters" (Horrigan, Boase, Rainie, and Wellman, 2006, p.2).

The report concludes, among other things, that the internet combined with other communication technologies improve and expand social networks and capital. This is not simply an elite phenomenon or relegated to the so-called developed world. For example, globally, mobile phones are the most common computing platform. Mobile adoption took 20 years (since 1983) to reach 1 billion subscribers, and only 3 years to double that number, with industry projections that this will increase to a billion subscribers a year (Romano, 2006).

The Pervasive Communication Environment Perspective 
Quite often, researchers find themselves in an either/or position, with any research that remotely touches on the Internet or mediated communication weighted down with general discourses on the nature of technology. To address this increasing cumbersome dichotomy I propose a perspective based on the Pervasive Communication Environment (PCE).

The PCE is founded on our multi-modal and trans-locational access to an integrated communications infrastructure that has text, audio, video, and voice capabilities. This fundamentally alters the dynamics of human interaction and enables dynamic and complex forms of cooperation and collective action. Much has been made of mobile communications, but it is important to recognize that the home is the foundation of the PCE. The variations of television, dial-up and broadband internet access, and even the humble land-line telephone make up a media and communication rich environment. Added to this are the more traditional mobile media - print and radio in their various forms. While these are normally one-way channels with individuals as receivers, they become multi-directional and interactive when combined with other technologies, especially new media devices. New media devices such as complex mobile technologies, WiFi enabled portable computers, and digital recording devices, link into a larger increasingly more integrated communication infrastructure broadly understood as the Internet. This digital infrastructure integrates older media forms, both mobile and fixed, into a larger pervasive communication environment. Of course, at its center is still the fundamental interaction and production of humans communicating with each other. All of these elements combine in an interactive dance with each node of the communication structure linked in a multitude of ways with every other node.

Based loosely on Hall's "Circuit of Communication" (1980) depicting culture and mass communication processes, the PCE model attempts to address the complex interactions and 
feedback loops that exist in much of the global north and is steadily expanding south. [Insert figure1.jpg here]

The schematic is overlaid on the communication infrastructure that is developing to explicitly (though actual direct wired or wireless connections) and implicitly (though reference or indirect connections) connect mobile and fixed media and communication sources. The outer ring is comprised of three general areas - fixed or generally location specific connections; mobile wireless devices; and mobile traditional media. The inner ring represents person-to-person collocated communication. The rings and the elements within them are then connected via complex and overlapping feedback loops. Of course, any model is a drastic oversimplification, since potentially any of the media sources can be placed with another area under specific circumstances, such as a portable TV or home use of WiFi (yours or a neighbors!) and mobiles.

\section{Outer Ring: Fixed/Location Specific Media}

This area is comprised of media that is generally interacted with in the context of a fixed physical location either at home, school, or work. This area highlights the home as the main media hub and recognizes that both dial-up and broadband connections are serviced through monopoly providers to fixed geographical locations. Public or commercial facilities that allow for access and provide computers are also fixed locations and would fall under this area. WiMax and mesh networks and other advanced connectivity schemes that collectively service entire areas might complicate this classification. Home VCR or audio (tape/CD) player/recorders would also be included. 


\section{Outer Ring: Mobile Digital Media}

This area reflects the proliferation of mobile wireless devices. Currently mobile broadband connectivity is primarily tied to laptop computers using some version of WiFi whose source is not controlled by the user. These can be intentionally or fortuitously open or pay-peruse. While access is location specific for WiFi signals, the user owns the terminal and can access many different networks as the computer is relocated. Mobile service enabled laptop connectivity would also fall within this area. The bulk of these devices are mobiles with various degrees of computing power. Of particular interest is the ability of these devices to acquire still photo, video, or audio and transmit them to other users mobiles, computers, or websites as well as receive such media. This is in addition to text and voice. Portable digital music players, such as the iPod, and digital cameras or video recorders, generally must be connected to a computer in order to share media. DVD/CD players are included because the ability to play and/or produce or burn content onto discs for later use. These devices, unlike multi-functional mobiles, tend to be task specific. These offer time and location shifting ability in easier to transport packages. It is important to note that many new generation devices, such as Apple's new iPhone will increasingly blur the distinction between these devices and further layers of complexity.

\section{Outer Ring: Print and Broadcast Radio}

Print publications, such as newspapers, magazines (and books for that matter) and broadcast radio receivers were the original portable media devices. Digital media does not obscure the clear dominance of these modes of consumption. While both of these categories can 
be consumed in a fixed location, their mobility and portability is a primary strength. Newspapers traditionally offer a limited degree of interactivity through letters to the editor, but increasingly are connected through websites that allow for interaction that is more robust. Likewise, telephony (first landline, then mobile) has allowed for more interaction with broadcast radio. In fact, the call-in radio talk show is a staple on commercial and non-commercial stations alike. Streaming audio, webcasting, and the advent of the podcast has increased radio's interaction with other media. Some stations, such as community station KEXP in Seattle WA, have leveraged their online presence and now command a global audience that not only listens but pledges. The proposed conversion to In-Band-on-Channel (IBOC) digital audio broadcasting in the US would also bring radio in-line with other wireless devices.

\section{Connecting the Outer Ring}

Much like Hall's (1980) model, the bi-directional arrows illustrate how all these different media/communication areas interact with each other both in production and consumption. Some examples would be Voice Over Internet Protocol (VOIP) between fixed and wireless computers, landlines, and mobiles; time and location shifting of media content between receivers and mobile devices; transferring content from portable capture devices to the web and the subsequent portability of such content to all media. And of course the dominance of print, television, and film content online. These are areas of connectivity that reflect the technical infrastructure.

Inner Ring: Person-to-Person

The inner ring represents face-to-face interaction by collocated individuals. Most of what we know about the outside world comes to us by way of media or with conversations conducted 
via media (from voice to physical letters). Our regular face-to-face interactions are a forum where we identify potential media sources, or content, or simply relay the information we have acquired. The inner ring also represents the human element in the novel connection of media and the methods employed by users to connect their media to each other. This is a distinct context because, unlike the outer ring that represents larger infrastructure and interoperability built into the communication structure, the inner-ring represents the novel ways that users mix and mash media and devices to suit their own ends (Thurlow and Brown, 2003). Two obvious examples are the rise of peer-to-peer music file sharing (computers, broadband, mp3, portable digital music players) and mobile phone cameras (used as cheap cameras instead of sending photo-augmented messages as was the intent). Novel uses often drive the market as much as large-scale infrastructure or format choices. This ring also represents direct basic person-to-person communication via devices (mainly voice and text). These conversations can also be linked to other media via the use of hypertext links or simple references to other media content. These interactions, in turn, flow outward into the broader media-sphere.

Floating Elements: Context, Environment, Feedback, Noise, and Channel

Some elements float and are concerned with broader issues of interaction and communication. These influence the quality, use, and choices individuals make in communication practices.

Context refers to factors that are not directly related to the specific act of communication. The actual space in which the individual is communicating influences the message, the response, and the mode of communication. For example, the use of text messaging is often related to situations in which speaking is not practical or desirable or if the sender wishes to limit 
synchronous communication.

The environment refers to external surroundings that influence communication such as broader cultural norms or socio-political situations. For example, some nations efforts to censor Internet content.

Feedback from others, both verbal and non-verbal, provides individuals with a sense of how others are interpreting them. This also allows individuals to choose or combine different communication modes. For example, text messaging during a meeting or switching to a mobile if the Internet cannot be accessed.

Noise refers to anything that interferes with communication. Noise may be internal to the individual (state of mind, physical distractions, inattentiveness, or miss-interpretation) or external (technical issues, connectivity, noise, other distractions).

Channel simply refers to the mode(s) or medium(s) of communication (in-person, print, or electronic). Channels are often combined.

Bi-directional arrows connecting inner and outer rings represent the inner-connectivity between the more personal interactions between individuals (mediated or face-to-face) and the broader production and consumption of media across various devices and networks. An example of this process is illustrated below. This diagram reflects the origins of the PCE model in my exploration of dissent network structures and information cascading through various nodes. [Insert Figure2.jpg]

In this example, a message is received and posted to a listserv where it is consumed and is passed along though multiple channels. Any path can lead to potentially any media source. 
Model Utility as an Analytical Framework

As envisioned, the PCE model has multiple applications for both research and pedagogy. Below I give a brief overview of possible applications in these two areas. The first is its adaptation to the teaching of public speaking. In communication, public speaking is referred to as "the basic course" and forms the foundation for many communication programs. Many colleges and universities require all undergraduates to take a public speaking course. This adaptation is from Public Speaking: The Evolving Art (S. Coopman \& Lull, 2009).

Public Speaking the Pervasive Communication Environment

[Insert figure3.jpg] The figure below is an adaptation of the PCE model for public speaking.

While maintaining the thematic design and most primary areas, it combines and collapses others, modifies some terms to fit the specific context, and adds elements that are specific to the topic area. This highlights the utility and flexibility of the model. For the purposes of student's audience analysis and research, the mass media is collapsed into one category regardless of source type. Distinct aspects of concern for public speaking, such as contextualizing the message and analysis of the speaking environment are added.

Context refers to the physical setting for a speech. The actual space in which the speaker talks influences the message and how the audience responds, as well as the occasion for the speech, contributes to the context.

The environment is the external surroundings that influence a public speaking event such as cultural norms, current events, and recent societal changes. 
Feedback from listeners, both verbal and non-verbal, provides speakers with a sense of how the audience is interpreting the message.

Noise refers to anything that interferes with listeners understanding the message. Noise may be internal to the listener (daydreaming, dismissing a speech as irrelevant or boring, thinking about something else, being hungry or tired) or external (sounds that prevent listeners from hearing, such as talking, a mobile phone ringing, or cars on a nearby street). Poor lighting and ineffective use of presentation media are also sources of noise (Coopman and Lull, 2009).

The model also differentiates between the speaker (main producer of the content) and the audience (as the consumer), rather than a general interactive approach of the PCE model. Channel (in this case) takes in to account the use of multimedia before, during, and after the speech (Coopman and Lull, 2009).

The utility of this adaptation becomes more apparent when examining a more traditional approach to public speaking where the focus is on speeches given in front of a live audience in a physical space. Again, this mode of face-to-face communication is considered by many instructors to be the standard against which all else is measured and competency in this limited realm is assumed to transfer to other venues. This idea is perpetuated in many textbooks. However, this traditional approach does not address even textual treatments of speeches, let alone the realities of web-cams, multimedia presentations, podcasting, video-conferencing, and a host of other applications the students will face on a daily basis. Use of this model as a teaching tool could assist in helping students to visualize the complex factors that make up the preparation as well as the act of public speaking.

Research Application: the Study of Social Movements 
The impetuous of this model is a result into my study of social movement's use of the Internet as a communication and organizing tool. In particular, the use by the micro radio movement whose goal is to increase public access to the radio spectrum in the US and the Independent Media Center (IMC) or Indymedia movement, which uses multimedia websites and citizen journalists to challenge mainstream media. For our purposes, an analysis of the IMC movement would provide a better example. Many Indymedia centers where started to support large-scale protest actions, the best example being in Seattle for the 1999 World Trade Organization (WTO) Ministerial Meeting. The Independent Media Center is a creature of the interface between portable media recorders and networks and is therefore a good test case for the PCE model.

Some IMCs have a physical location with computers and other multimedia equipment available while other exist "virtually" as websites on servers scattered around the globe. These websites can be accessed via any Internet connection, with high-level access controlled by password protection. Indymedia Centers are geographically grounded in the communities they serve. Some collectives are self-contained in their media production, while others partner with other media in different mediums. For example, offering a link to the streaming webcast of a local pirate/free radio station or producing a newspaper. All sites allow for uploading of multimedia by the general public (with restrictions varying among collectives) which account for a majority of website content. For this analysis I begin moving from the inner ring out. IMC: Inner Rings

Indymedia Centers are built around collectives of activists organized on a radical democratic or consensus decision-making model. Physical face-to-face meetings take place in small working groups and the full collective. These collectives and groups are, in turn, linked via 
listservs and/or instant messaging. Groups usually have specific responsibilities such as maintaining the code running the website, editorial content, or production of specific mediums such as video, radio, or print publication.

IMC: Fixed Media

IMC's with physical locations have public access computers and websites allow access and uploading from any Internet enabled machine in any location. Indymedia content is often shared across the network and more organized IMC's produce an IndyNews Reel program of website or original video that is presented via public access cable or similar television outlets as well as being shared via the Deep Dish satellite network.

IMC: Mobiles

Mobiles are heavily used to coordinate journalists in the field and to give updates to those with access to the websites on news and events. In some cases, texting and other phone enabled applications can be directly linked to an Indymedia site for uploading. Using WiFi access would be the same as fixed access and some IMC physical locations have WiFi capability. The main utility would be the ability to upload multimedia from public or commercial WiFi hotspots on developing events such as protests. This would allow for quick access to the Indymedia sites to post breaking news. Perhaps the biggest boon to the production of IMC content is the increased availability of portable digital recording devices. Digital video and photography as well as digital recorders have made newsgathering and sharing increasing easy. Even analog tape machines can be converted to digital for upload with the right equipment.

IMC: Print and Radio

Many IMCs run print newspaper operations either on a regular basis or for major events. The content for these papers is often from the Indymedia website as well as original content. Of 
course, these papers are composed via computers. Many IMCs also have their own webcasting operations or work in conjunction with pirate or community radio stations to provide audio available through the website, links to other websites, and the band frequency of broadcasts. Audio content is often exchanged between sources.

\section{Conclusion}

The pervasive communication environment (PCE) perspective and model reflects the emerging infosphere and its complexity in a basic figure. The PCE perspective offers a holistic analytical approach to our multi-modal and trans-locational access to an integrated communications infrastructure that has text, audio, video, and voice capabilities. These capabilities alter the dynamics of human interaction and enables dynamic and complex forms of cooperation and collective action. It takes into account the communication imperative (Thurlow and Brown, 2003; Thurlow et al, 2004) that underlies all human interaction. The variations of television, dial-up and broadband Internet access, and land-line telephones and the more traditional mobile media -print and radio in their various forms make up a media and communication rich environment. While many of these are normally one-way channels with individuals as receivers, they become multi-directional and interactive when combined with new media devices. New media devices such as complex mobile technologies, WiFi enabled portable computers, and digital recording devices, feed into a larger and more integrated communication infrastructure clustered around the Internet. This digital infrastructure integrates existing media forms, both mobile and fixed, into a larger pervasive communication environment. As with most communication, at its center is the fundamental interaction and production of humans communicating. All of these elements combine interactively with each node of the 
communication structure linked in a multitude of ways with every other node. By utilizing the PCE perspective, scholars may find novel and important aspects of their research that might otherwise have been occluded by a narrower approach. For instructors, PCE perspective assists in helping students to visualize our complex social environment as well as understanding how communication technology shapes everyday communication practices. 


\section{References}

Althaus, S., 1997. “Computer Mediated Communication in the University Classroom: An Experiment with On-line Discussions." Communication Education, volume 46, pp. 158-174.

Alexander, C., Ishikawa, S., Silverstein, M., Jacobson, M., Fiksdahl-King, I., and Angel, S., 1977. A Pattern Language. New York: Oxford University Press.

An, Y.J., and Frick, T., 2006. "Student Perceptions of Asynchronous Computer-Mediated Communication in Face-To-Face Courses." Journal of Computer-Mediated Communication, volume 1, issue 2 at http://jcmc.indiana.edu/vol11/issue2/an.html accessed on July 30, 2006.

Benoit, P.J., Benoit, W.L., Milyo, J, and Hanson, G.J., 2006. The effects of traditional vs. web assisted instruction on students learning and satisfaction (August). Andrew Mellon Foundation Report. Columbia, Missouri: University of Missouri.

Brandon, D., and Holligshead, A., 1999. "Collaborative Learning in Computer Supported Groups.” Communication Education, Volume 48, pp. 109-126.

Bordia, P., 1997. Face-To-Face Versus Computer-Media Communication: A Synthesis of the Experimental Literature. Journal of Business Communication, Volume 34, pp. 99-120.

Coopman, S. J., \& Lull, J., 2009. Public speaking for the digital age. Belmont, CA: Wadsworth 
Hall, S., 1980. “Encoding/decoding.” In S. Hall, D. Hobson, A. Lowe, \& P. Willis, (Eds.), Culture, Media, Language. London: Routledge, pp. 128-138

Horrigan, J.B., Boase, J., Rainie, L., and Wellman, B., 2006. "The Strength Of Internet Ties: The Internet and Email Aid Users in Maintaining their Social Networks and Provide Pathways to Help when People Face Big Decisions." Pew Internet and American Life Project at http://www.pewinternet.org/ accessed on January 25, 2006

Kim, Y. and Ball-Rokeach, S. J., 2006. Civic Engagement from a Communication Infrastructure Perspective. Communication Theory volume 16 issue 2, pp. 173-197.

Pang, A. S. and Pescovitz, D., 2006. “Cyberspace is dead.” Wired 14.02 (February), p.39.

Papacharissi, Z., 2005. “The Real-Virtual Dichotomy in Online Interaction: New Media Uses and Consequences Revisited.” In P. Kalbfleisch (Ed.), Communication Yearbook 29. Mahwah, NJ: Lawrence Erlbaum. pp. 215-237.

Postmes, T., Spears, R., and Lea, M., 2000. “The Formation of Group Norms in Computer-Mediated Communication.” Human Communication Research. volume 26 (3, July), pp. 341-371.

Rafaeli, S., 1988. "Interactivity: From New Media to Communication.” In R. P. Hawkins, J. M. Wiemann, and S. Pingree (eds.). Advancing Communication science: Merging Maps and Interpersonal Processes. Newbury Par, CA: Sage. pp. 110-134 
Ramirez, Jr., A., Walther, J., Burgoon, J., and Sunnafrank, M., 2002. "Information-Seeking Strategies, Uncertainty, and Computer-Mediated Communication Toward a Conceptual Model." Human Communication Research. volume 28 (2, April), pp. 213-228.

Romano, B. J., 2007. “Motorola's Calling: It's a Mobile world.” The Seattle Times (January 9), pp. E-1, E4.

Thurlow, C. and Brown, A., 2003. "Generation txt? The Discourses of Young People TextMessaging." Discourse Analysis Online

http://extra.shu.ac.uk/daol/articles/v1/n1/a3/thurlow2002003-01.html accessed on December 13, 2006

Thurlow, C., Lengel, L. and Tomic, A., 2004. Computer Mediated Communication: Social Interaction and the Internet. London: Sage.

Walther, J.B., Gat, G., and Hancock, J. T., 2005. "How Do Communication and Technology Researchers Study the Internet?” Journal of Communication volume 55, issue 3. pp. 632-657.

Wellman, B., 2001. “Computer Networks As Social Networks.” Science (September) volume 293, pp. 2031-2034.

Wellman, B., 2000. “Changing Connectivity: “A Future History of Y2.03K.” Sociological ResearchOnline, volume 4, at http://www.socresonline.org.uk/4/4/wellman.html accessed on 
June 1, 2003.

\begin{abstract}
About the Author
Ted M. Coopman (Ph.D. Communication, University of Washington, 2008) is a lecturer in the Department of Communication Studies and the Department of Television, Radio, Film, and Theatre at San José State University, San José CA.
\end{abstract}

\title{
Copyright Statement
}

This article may be used for non-commercial purposes with attribution. Requests for commercial use should be directed to the author and must be obtained in writing. 